ELECTRONIC SUPPLEMENTARY MATERIAL

\title{
Layer-by-Layer Assembly of Carbon Dots-Based Ultrathin Films with Enhanced Quantum Yield and Temperature Sensing Performance
}

\author{
Wendi Liu, Simin Xu, Zhixiong Li, Ruizheng Liang*, Min Wei*, David G. Evans, and \\ Xue Duan
}

State Key Laboratory of Chemical Resource Engineering, Beijing University of Chemical Technology, Beijing 100029, P. R. China

${ }^{*}$ Corresponding authors. Tel: +86-10-64412131; Fax: +86-10-64425385.

E-mail addresses: liangruizheng2000@163.com (R. Liang); weimin@mail.buct.edu.cn (M. Wei). 


\section{Experimental Section}

\section{Materials}

Analytical pure $\mathrm{Mg}\left(\mathrm{NO}_{3}\right)_{2} \cdot 6 \mathrm{H}_{2} \mathrm{O}, \mathrm{Al}\left(\mathrm{NO}_{3}\right)_{3} \cdot 9 \mathrm{H}_{2} \mathrm{O}$, citric acid, ethylenediamine and urea were purchased from Beijing Chemical Co. Ltd. and used without further purification. Poly (styrene 4-sulfonate) (PSS, Mw=7,000) was purchased from Sigma Chemical Co. Ltd. The deionized and decarbonated water was used in all the experimental processes.

\section{Preparation of carbon dots}

Carbon dots (CDs) were prepared according to the reported method. ${ }^{1}$ Citric acid (1.000 g) and ethylenediamine $(335 \mu \mathrm{L})$ were dissolved in deionized water $(10 \mathrm{~mL})$, followed by a heating treatment at $200{ }^{\circ} \mathrm{C}$ for $5 \mathrm{~h}$ in a teflon-lined autoclave $(20 \mathrm{~mL})$. After the reaction, the autoclave was cooled to room temperature. Then the formative brown product was further dialyzed so as to obtain the purified CDs.

\section{Fabrication of (CDs@PSS/LDH)n UTFs}

Typically, $1.000 \mathrm{~g}$ PSS and $0.100 \mathrm{~g}$ CDs were dissolved in $100 \mathrm{~mL}$ of water to form CDs@PSS colloidal solution. The synthesis and exfoliation of MgAl-LDH were similar to that described in our previous work. $^{2} 0.100 \mathrm{~g}$ of MgAl-LDH was shaken in $100 \mathrm{ml}$ of formamide solution for $48 \mathrm{~h}$ to produce a colloidal suspension of exfoliated MgAl-LDH nanosheets. The quartz glass substrate was firstly cleaned in concentrated $\mathrm{H}_{2} \mathrm{SO}_{4}-30 \% \mathrm{H}_{2} \mathrm{O}_{2}$ (7:3) for 30 min, followed by another $30 \mathrm{~min}$ in ethanol to remove the possible organics. After that, the quartz substrate was rinsed and washed thoroughly with deionic water. The substrate was then dipped in a colloidal suspension $\left(1 \mathrm{~g} \mathrm{dm}^{-3}\right)$ of LDH nanosheets for $10 \mathrm{~min}$ followed by thoroughly washing, and then the substrate was treated with 100 mL of CDs@PSS aqueous solution for 10 
min. Multi-layer films of $(\mathrm{CDs} @ \mathrm{PSS} / \mathrm{LDH})_{n}$ were fabricated by alternate deposition for $n$ cycles. The resulting films were dried under a nitrogen gas flow for 2 min.

\section{Cyclic Voltammetry of CDs}

To estimate the HOMO and LUMO energy levels of CDs, the cyclic voltammetry was performed using a three-electrode system. The counter electrode was platinum wire, and the reference electrode was $\mathrm{Ag} / \mathrm{AgCl}$. The working electrode was prepared by drop-casting $10 \mu \mathrm{L}$ CDs solution onto a platinum electrode followed by drying at $40{ }^{\circ} \mathrm{C}$. The electrolyte was 0.1 mtetrabutylammonium hexafluorophosphate (TBAPF6, Aldrich) in acetonitrile and was purged with $\mathrm{N}_{2}$ for 20 min prior to use. ${ }^{3}$

\section{Structural modeling of LDH system}

Model of MgAl-LDH was constructed according to the powder X-ray diffraction data. The space group of MgAl-LDH is $r \overline{3} m$, with the unit cell parameters $\alpha=\beta=90^{\circ}$, and $\gamma=120^{\circ}$. The molar ratio of $\mathrm{Mg}^{2+}$ to $\mathrm{Al}^{3+}$ is set to be 2 . The supercell of $\mathrm{MgAl}-\mathrm{LDH}$ is $3 \times 3 \times 1$ in the $a-, b$-, and $c$-direction. The nitrate anion is introduced into the interlayer space of MgAl-LDH to keep the model neutral.

All the calculations are performed with the CASTEP code in the Materials Studio, version 6.1 software package (Accelrys software Inc., San Diego, CA). ${ }^{4}$ The DFT calculations are performed using a plane wave implementation ${ }^{5}$ at the generalized gradient approximation (GGA) Perdew-Burke-Ernzerhof (PBE) level. ${ }^{6}$ The structure optimization is based on the following points: (1) an energy tolerance of $1 \times 10^{-5} \mathrm{eV} /$ atom; (2) a maximum force tolerance of $0.03 \mathrm{eV} / \AA$; and (3) a maximum displacement tolerance of $1 \times 10^{-3} \AA$. For the calculation of band structure of MgAl-LDH, the $\Gamma$-point-centered $k$-point meshes used for the Brillouin zone integrations are $3 \times 3 \times 1 k$-points. 


\section{Temperature-responsive performances in PL and ECL}

PL temperature response was performed by recording the PL spectra of the UTFs in the temperature range $20-80{ }^{\circ} \mathrm{C}$. ECL signal responses were tested by using a three-electrode system, including a platinum foil as the auxiliary electrode and a saturated $\mathrm{Ag} / \mathrm{AgCl}$ as the reference electrode. The working electrode was prepared by LBL assembling the $(\mathrm{CDs} @ \mathrm{PSS} / \mathrm{LDH})_{36} \mathrm{UTF}$ on the ITO glass. The ECL signals were recorded by a MPI-B multifunctional chemiluminescent analytical system (Remax Electronic Co. Ltd., Xi'an, China) with the voltage of the photomultiplier tube (PMT) at $800 \mathrm{~V}$. Temperature-dependent ECL signals were measured in the electrolyte at different temperatures $\left(20,50\right.$ and $\left.80{ }^{\circ} \mathrm{C}\right)$.

\section{Sample Characterization}

The UV-vis absorption spectra were collected in the range from 200 to $600 \mathrm{~nm}$ on a Shimadzu U-3000 spectrophotometer, with the slit width of $1.0 \mathrm{~nm}$. The fluorescence spectra were performed on a RF-5301PC fluorospectrophotometer with the excitation wavelength of $370 \mathrm{~nm}$. The fluorescence emission spectra are in the range from 390 to $600 \mathrm{~nm}$, and both the excitation and emission slit are set to $3.0 \mathrm{~nm}$. The Fourier transform infrared (FT-IR) spectra were recorded by a Nicolet 605 XB FT-IR spectrometer in the range $4000-400 \mathrm{~cm}^{-1}$ with $2 \mathrm{~cm}^{-1}$ resolution. Zeta potential was conducted with photon correlation spectroscopy (PCS, Nanosizer Nano ZS, MALVERN Instruments). The morphology of UTFs was investigated by using a scanning electron microscope (SEM Hitachi S-3500) equipped with an EDX attachment (EDX Oxford Instrument Isis 300), and the accelerating voltage applied was $20 \mathrm{kV}$. X-ray diffraction pattern (XRD) of the CDs@PSS/LDH UTF was recorded using a Rigaku 2500VB2+PC diffractometer under the conditions: $40 \mathrm{kV}, 50 \mathrm{~mA}, \mathrm{Cu} \mathrm{K \alpha}$ radiation $(\lambda=0.154056 \mathrm{~nm})$ with step-scanned in step of $0.04^{\circ}(2 \theta)$ in the range from 0.5 to $10^{\circ}$ using a count time of $10 \mathrm{~s} / \mathrm{step}$. 
The color coordinates of the fluorescence were determined by a PR-650 spectrocolorimeter. The surface distribution and thickness data were obtained by using the NanoScope IIIa atomic force microscope (AFM) from Veeco Instruments. TEM images were recorded on a JEOL JEM-2100 transmission electron microscope with the accelerating voltage of $200 \mathrm{KV}$. Photoluminescence quantum yield (PLQY) was measured using a Nanolog FL3-2iHR infrared fluorescence spectrometer equipped with an integrating sphere. The fluorescence decays were measured using LifeSpec-ps spectrometer with $370 \mathrm{~nm}$ laser excitation, and the lifetimes were calculated with the F900 Edinburgh instruments software. 

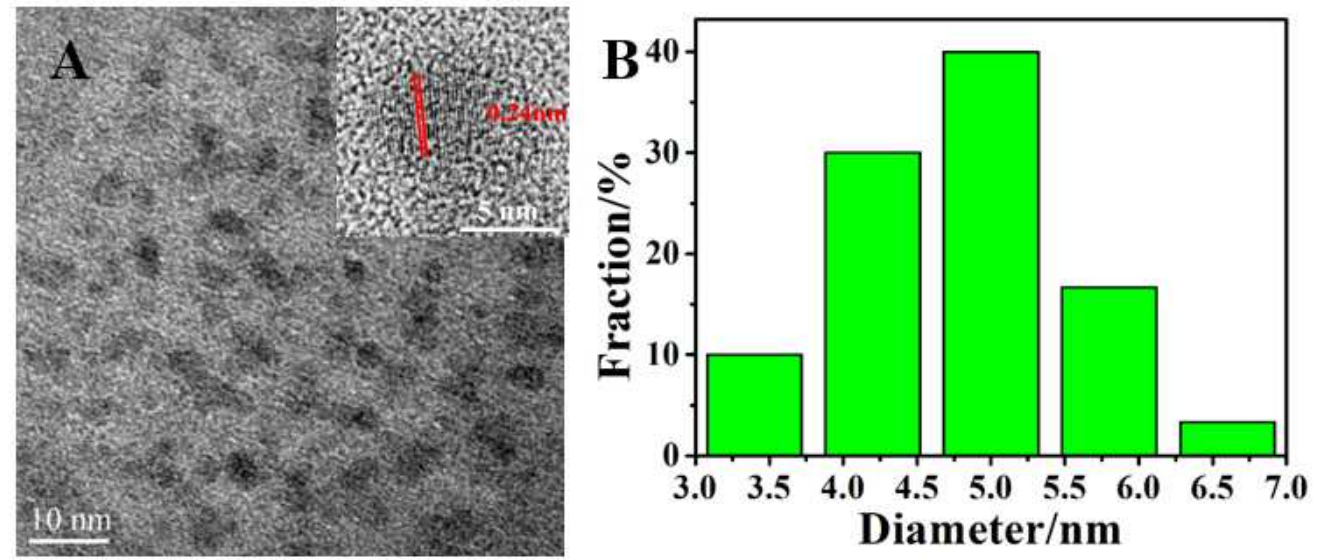

Figure S1. (A) HRTEM image and (B) particle size distribution of the as-prepared CDs. The inset in A shows the HRTEM image of a single particle with high magnification.
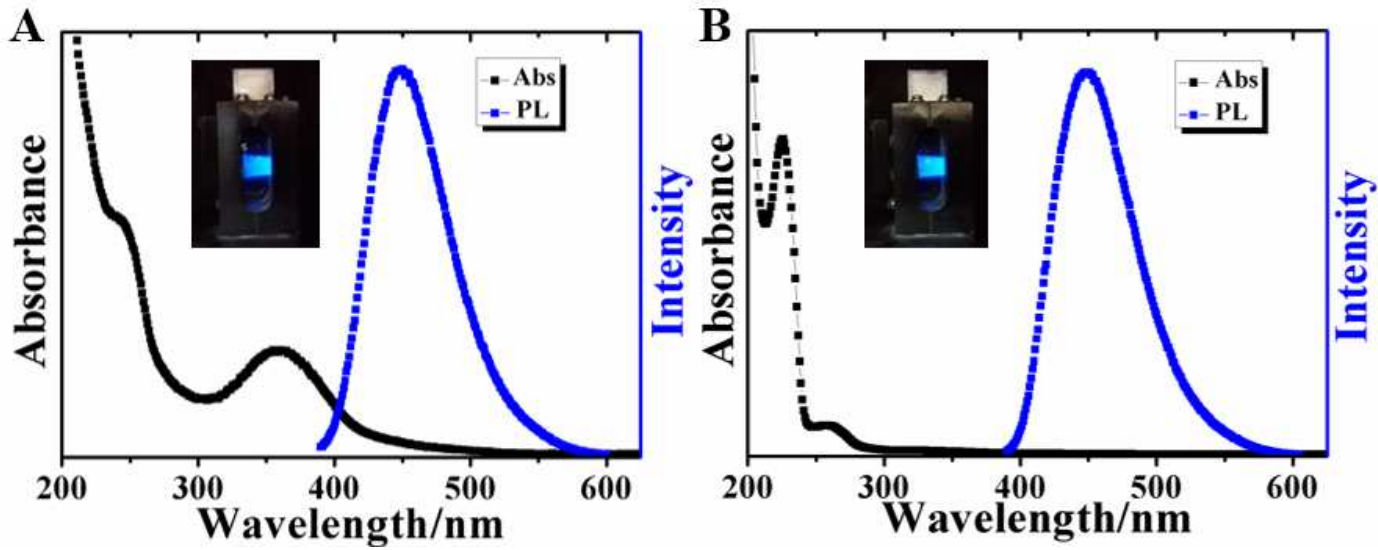

Figure S2. UV-vis absorption spectra, fluorescence spectra and photographs under UV light (370 nm, inset) of (A) pristine CDs solution and (B) CDs@PSS solution. 


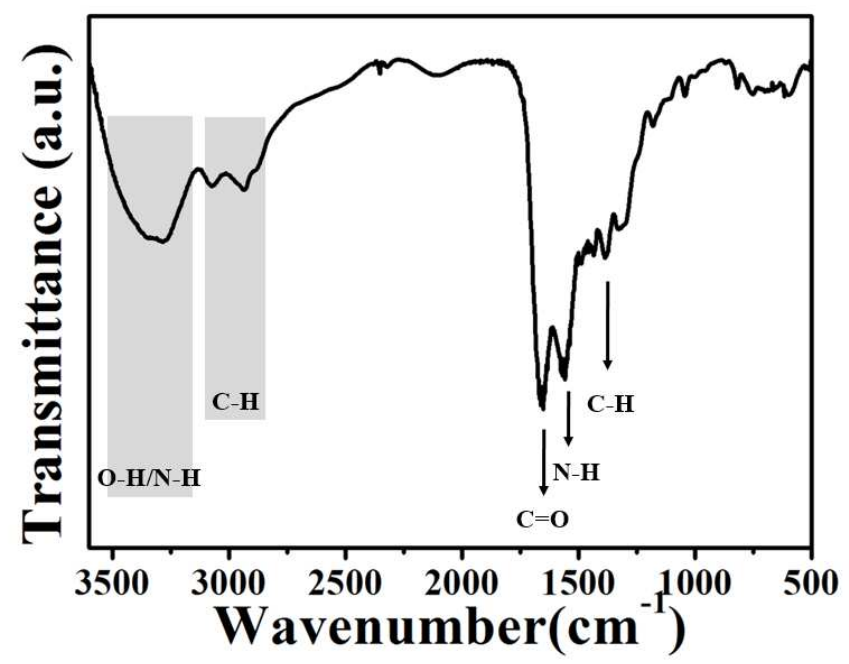

Figure S3. FT-IR spectrum of CDs.

The surface groups in the CDs can be confirmed by the FT-IR spectrum, such as stretching vibrations at $3200-3500,2850-3100$ and $1658 \mathrm{~cm}^{-1}$ (assigned to $-\mathrm{OH}$ or $-\mathrm{NH},-\mathrm{CH},-\mathrm{C}=\mathrm{O}$ ) as well as bending vibrations at 1560 and $1382 \mathrm{~cm}^{-1}$ (assigned to $-\mathrm{NH}$ and $-\mathrm{CH}$ ). These characteristic absorption bands indicate the existence of carbonyl, amino and hydroxyl groups on the surface of CDs.
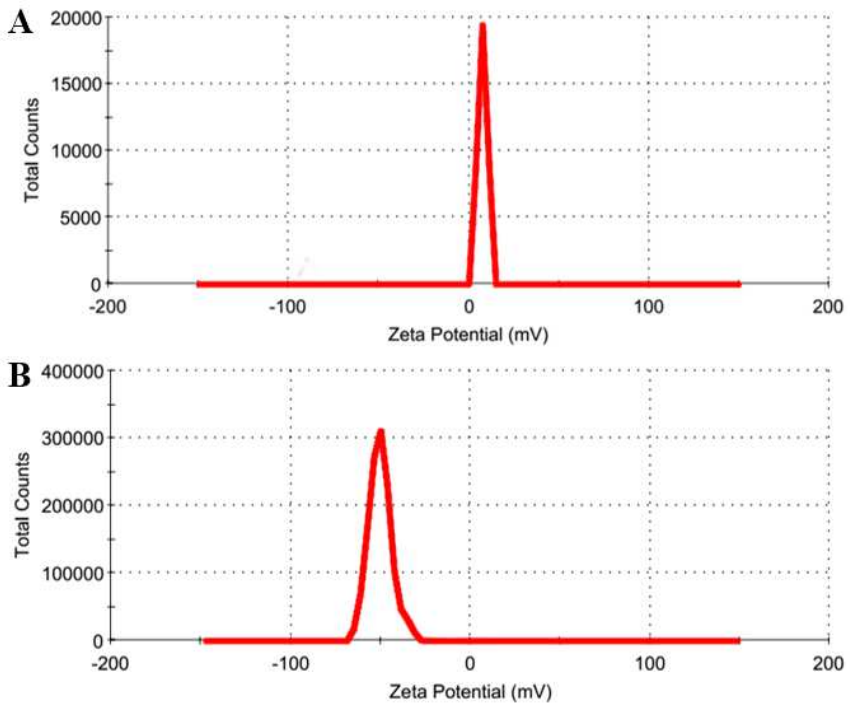

Figure S4. Zeta potential distribution of (A) CDs and (B) CDs@PSS. 

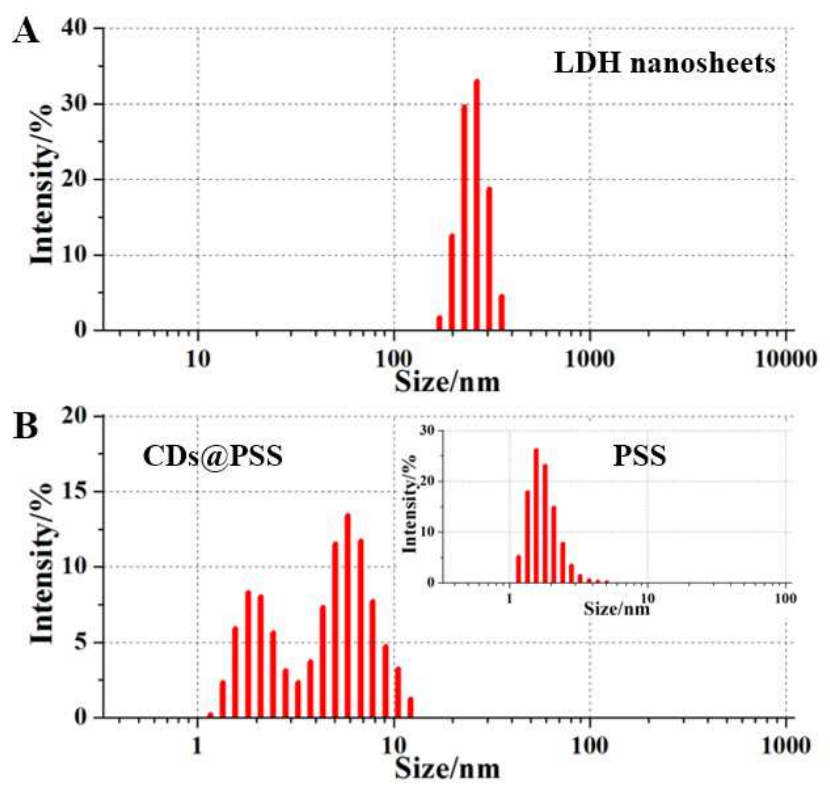

Figure S5. Size distribution of (A) exfoliated MgAl-LDH nanosheets and (B) CDs@PSS particles in colloid solution. The size of 1-3 nm in the CDs@PSS colloid solution is attributed to redundant PSS (inset in B).
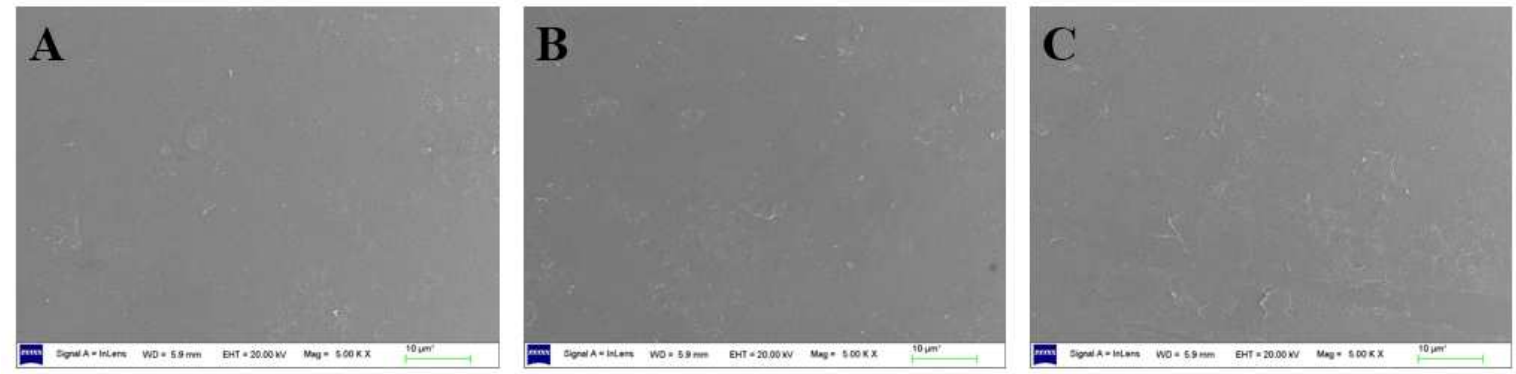

Figure S6. Top-view SEM images of the (CDs@PSS/LDH $)_{n}$ UTFs $(n=12,24,36$, respectively).
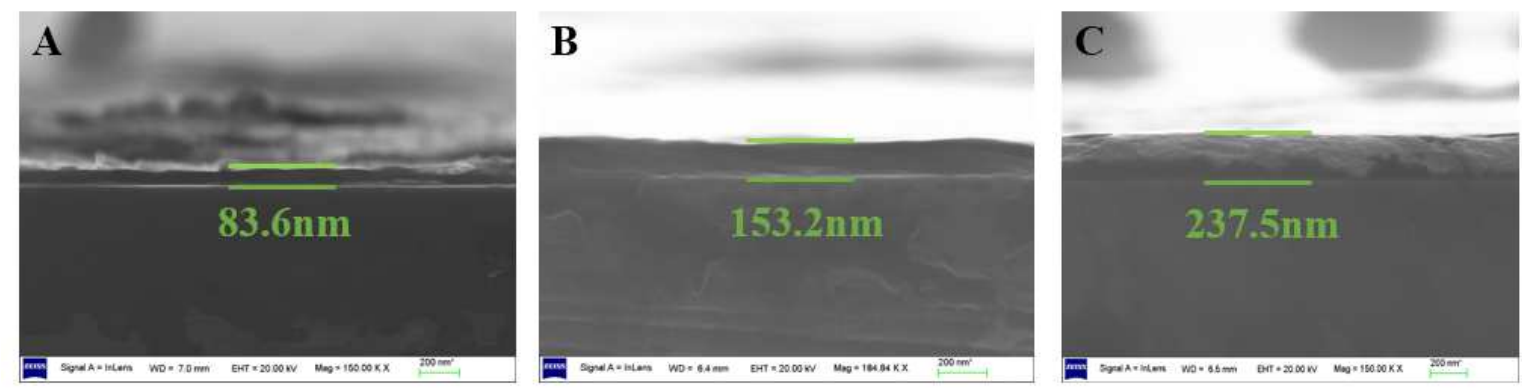

Figure S7. Side-view SEM images of the (CDs@PSS/LDH $)_{n}$ UTFs $(n=12,24,36$, respectively). 


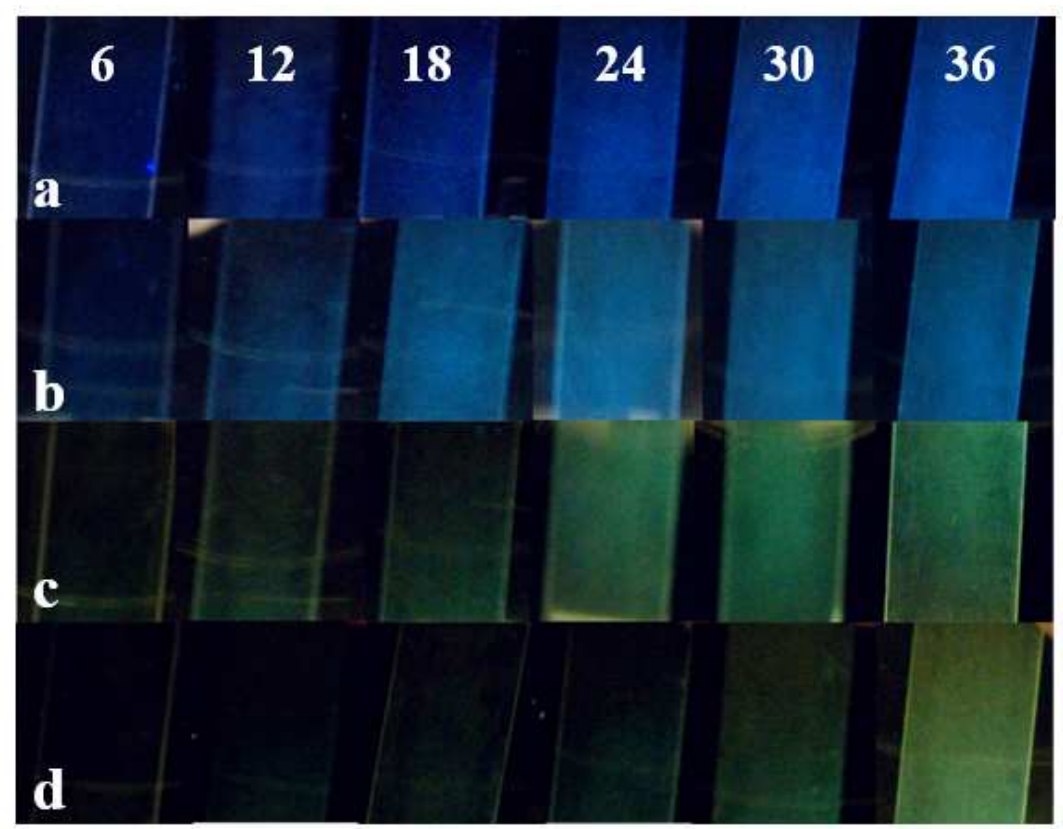

Figure S8. Photographs (taken under an RFPC light source) of the (CDs@PSS/LDH) ${ }_{n}$ UTFs (n=6-36) with different excitation wavelengths: (a) $370 \mathrm{~nm}$, (b) $400 \mathrm{~nm}$, (c) $430 \mathrm{~nm}$, (d) 460 nm.

Table S1. The double-exponential fitting of fluorescence decay data of (CDs@PSS/LDH) $)_{36}$ UTF and other control samples

\begin{tabular}{ccccc}
\hline Sample & $\tau_{i}(\mathrm{~ns})^{[\mathrm{a}]}$ & $A_{i}(\%)$ & $<\tau>(\mathrm{ns})$ & $\chi^{2[\mathrm{~b}]}$ \\
\hline CDs drop-casted film & 0.3158 & 61.38 & 0.813 & 1.861 \\
& 1.603 & 38.62 & & \\
CDs@PSS drop-casted & 4.503 & 30.54 & 9.398 & 1.183 \\
film & 11.55 & 69.46 & & \\
& 1.683 & 4.35 & 14.447 & 1.074 \\
(CDs@PSS/LDH) ${ }_{36} \mathrm{UTF}$ & 15.05 & 95.65 & & \\
\hline
\end{tabular}

[a] $\tau_{i}(i=1,2)$ is the fitted fluorescence lifetime. $A_{i}$ is the percentage of $\tau_{i}$. In this case, $<\tau>=A_{1} \tau_{1}+A_{2} \tau_{2} ; A_{1}+$ $A_{2}=1$. [b] The goodness of fit is indicated by the value of $\chi^{2}$. 

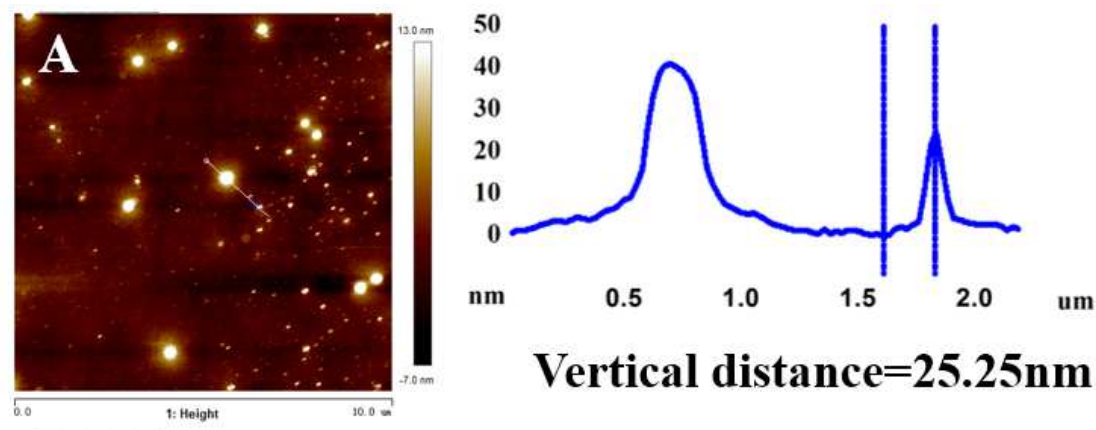

\section{Vertical distance $=25.25 \mathrm{~nm}$}
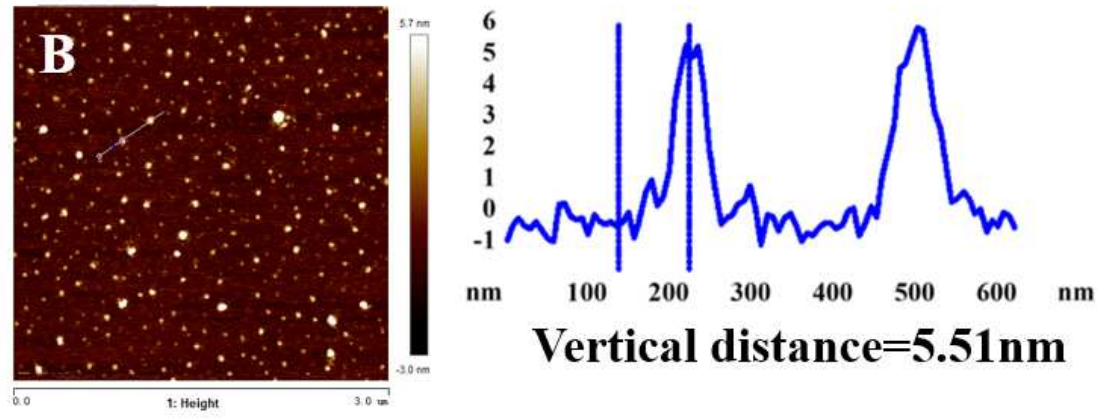

Figure S9. AFM images and corresponding height profiles of (A) CDs drop-casted film and (B) single layer CDs@PSS/LDH UTF.

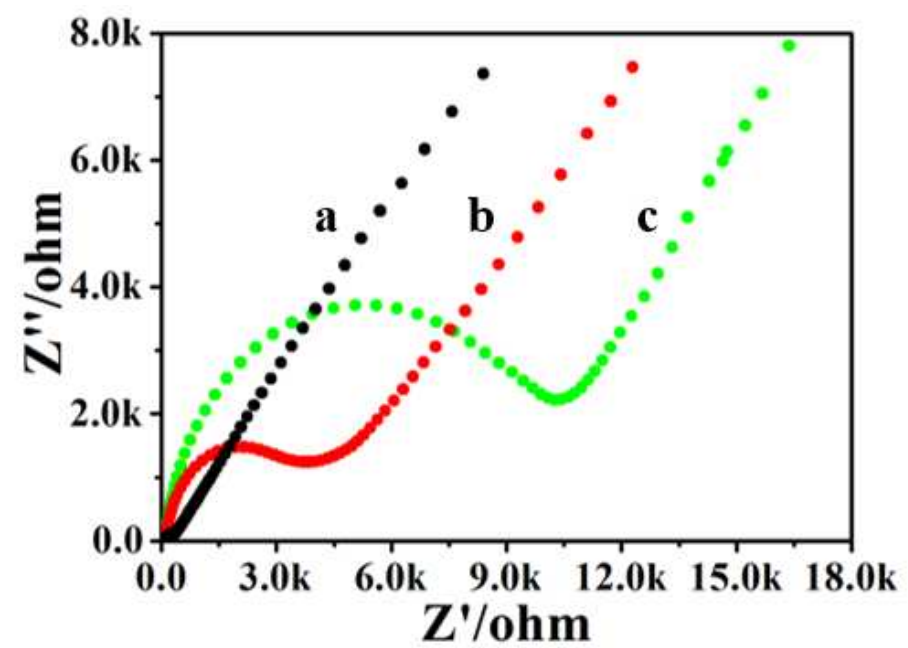

Figure S10. Nyquist plots of the electrochemical impedance spectroscopy of (a) CDs, (b) CDs@PSS and (c) CDs@PSS/LDHs. 

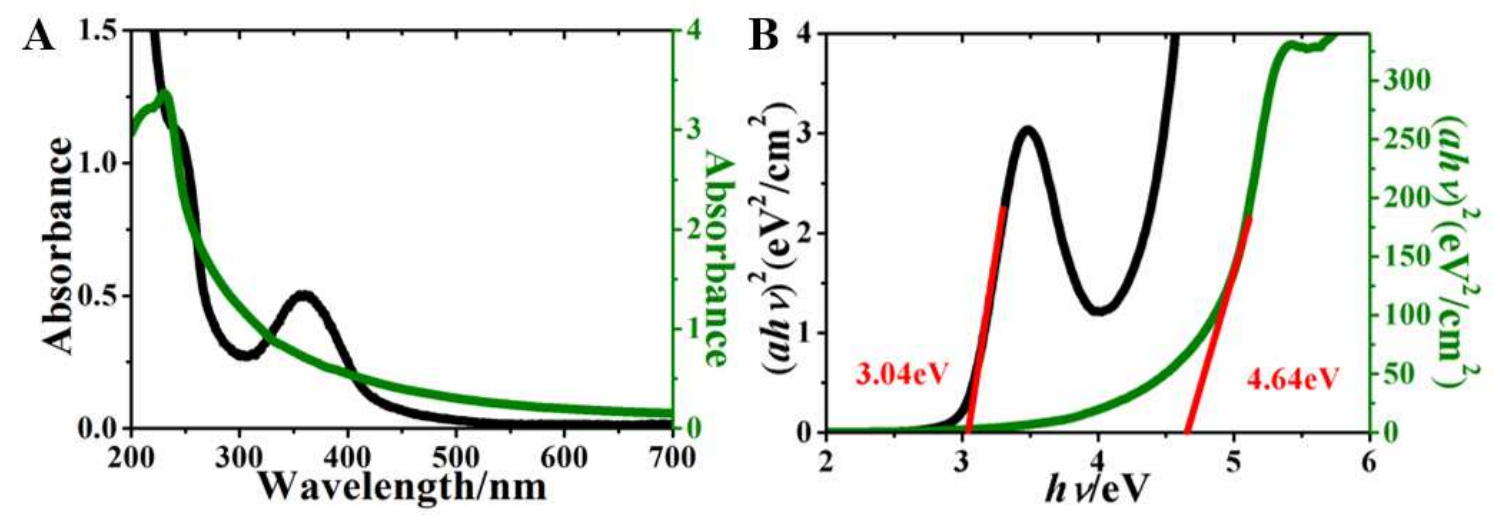

Figure S11. (A) UV-vis absorption spectra and (B) absorption threshold of CDs (black line) and MgAl-LDH (green line).
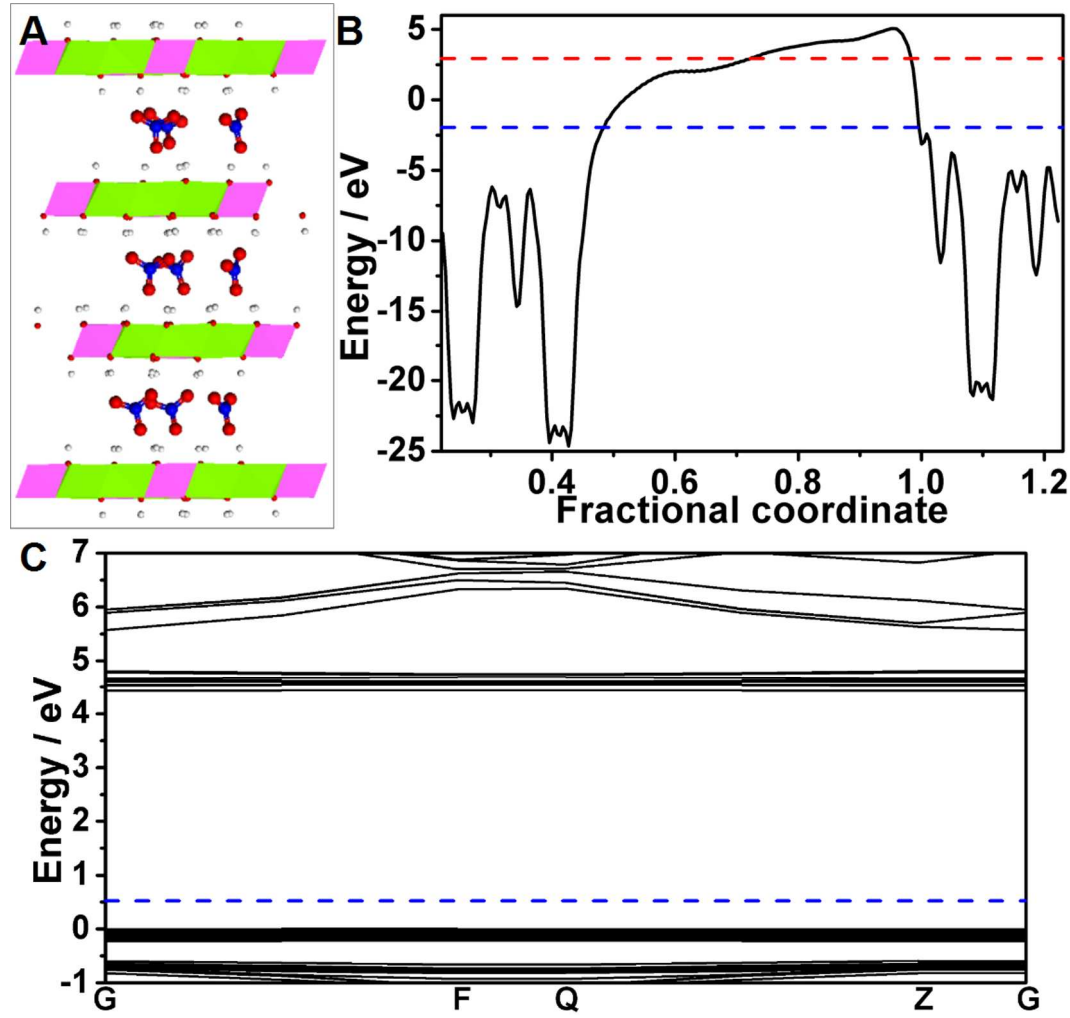

Figure S12. (A) The optimized geometry, (B) the work function and (C) the band structure of MgAl-LDH. The dashed red and blue line in (B) represent the vacuum level and Fermi level, respectively. 

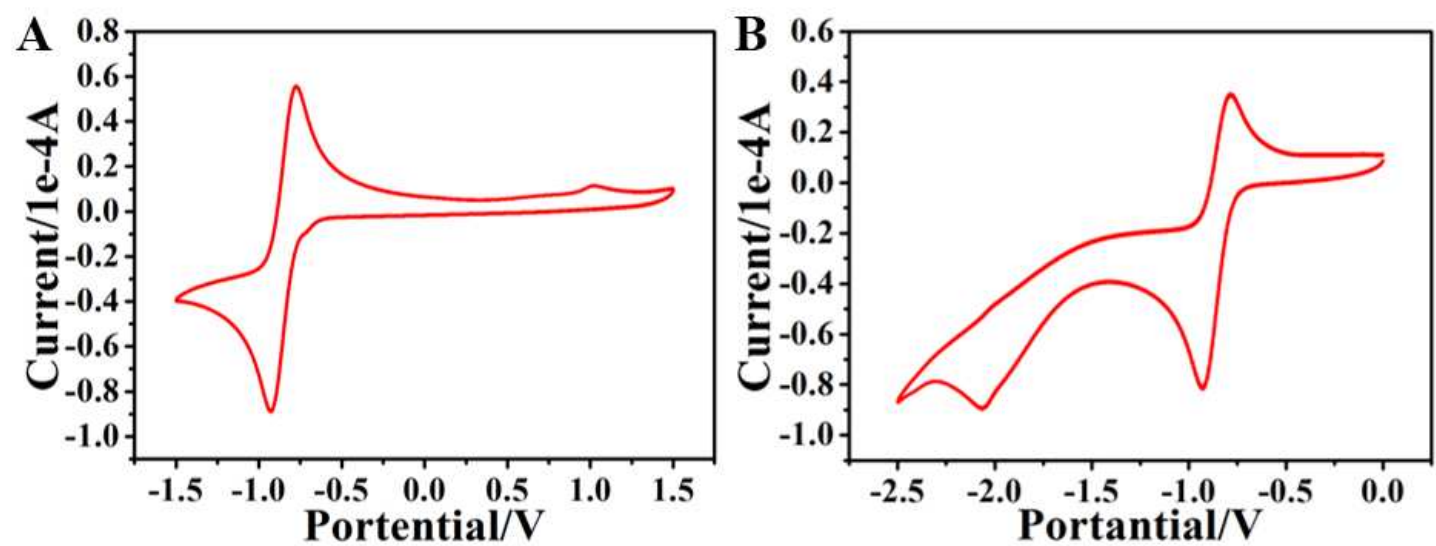

Figure S13. The cyclic voltammogram curves of CDs: (A) the positive scanning, (B) the negative scanning.

The HOMO and LUMO energy levels of CDs were calculated according to the following equations:

$$
\begin{aligned}
& E_{(\text {номо })}=-\mathrm{e}\left(E_{\text {ox }}+4.4\right)(\mathrm{eV}) \\
& E_{(L U M O)}=-\mathrm{e}\left(E_{r e d}+4.4\right)(\mathrm{eV})
\end{aligned}
$$

Where $E_{o x}$ and $E_{r e d}$ are the onset of oxidation and reduction potential, respectively. $E_{o x}$ and $E_{r e d}$ were determined to be 1.02 and $-2.06 \mathrm{~V}$ and the corresponding LUMO and HOMO levels were -5.42 and $-2.34 \mathrm{eV}$, respectively.

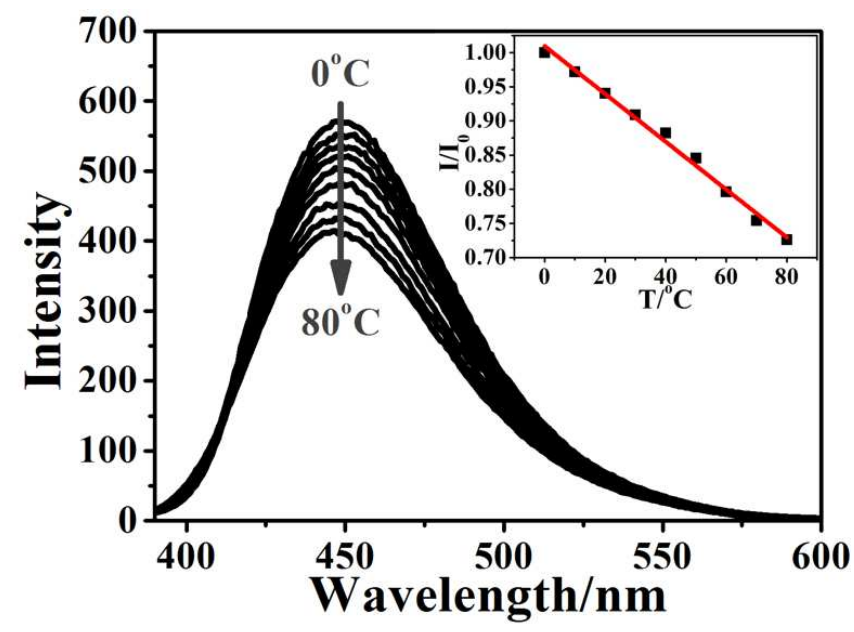

Figure S14. Luminescence spectra of CDs aqueous solution in the temperature range $0-80{ }^{\circ} \mathrm{C}$ (inset: the relative emission intensity as a function of temperature). 
Table S2. The double-exponential fitting of fluorescence decay data of (CDs@PSS/LDH $)_{36}$ $\mathrm{UTF}$ at 20 and $80^{\circ} \mathrm{C}$

\begin{tabular}{ccccc}
\hline $\mathrm{T} /{ }^{\circ} \mathrm{C}$ & $\tau_{i}(\mathrm{~ns})^{[\mathrm{a}]}$ & $A_{i}(\%)$ & $<\tau>(\mathrm{ns})$ & $\chi^{2[\mathrm{~b}]}$ \\
\hline 20 & 1.683 & 4.35 & 14.447 & 1.074 \\
& 15.05 & 95.65 & & \\
80 & 1.114 & 30.22 & 10.87 & 1.313 \\
& 15.1 & 69.78 & &
\end{tabular}

[a] $\tau_{i}(i=1,2)$ is the fitted fluorescence lifetime. $A_{i}$ is the percentage of $\tau_{i}$. In this case, $<\tau>=A_{1} \tau_{1}+A_{2} \tau_{2} ; A_{1}+$ $A_{2}=1$. [b] The goodness of fit is indicated by the value of $\chi^{2}$.

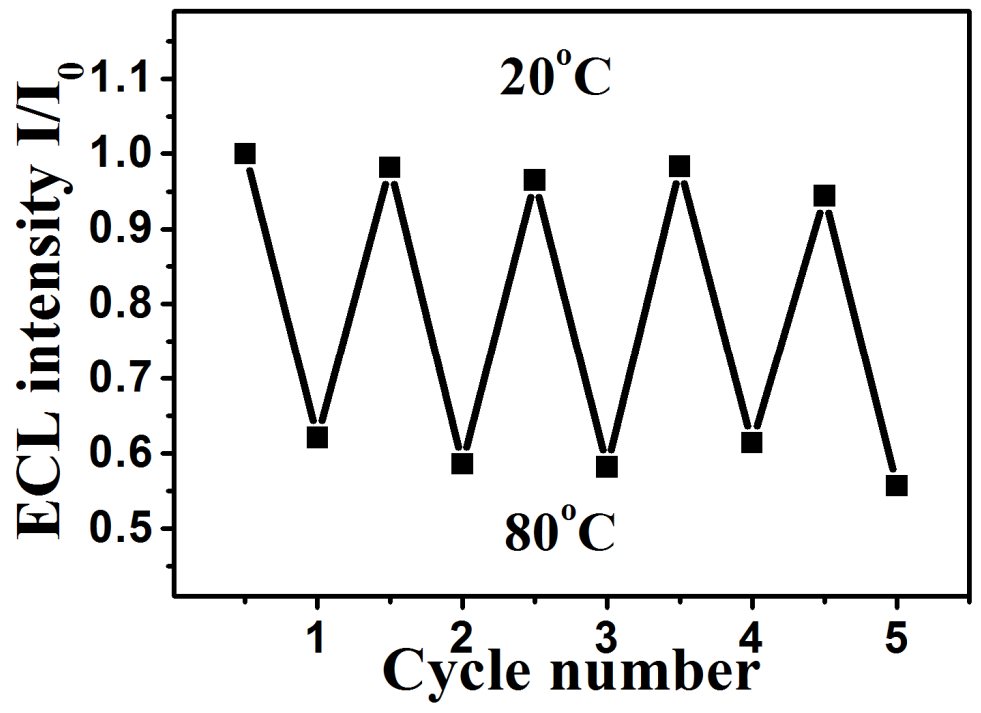

Figure S15. Reversibility test of ECL intensity for the (CDs@PSS/LDH) $)_{36}$ UTF at 20 and 80 ${ }^{\circ} \mathrm{C}$. 


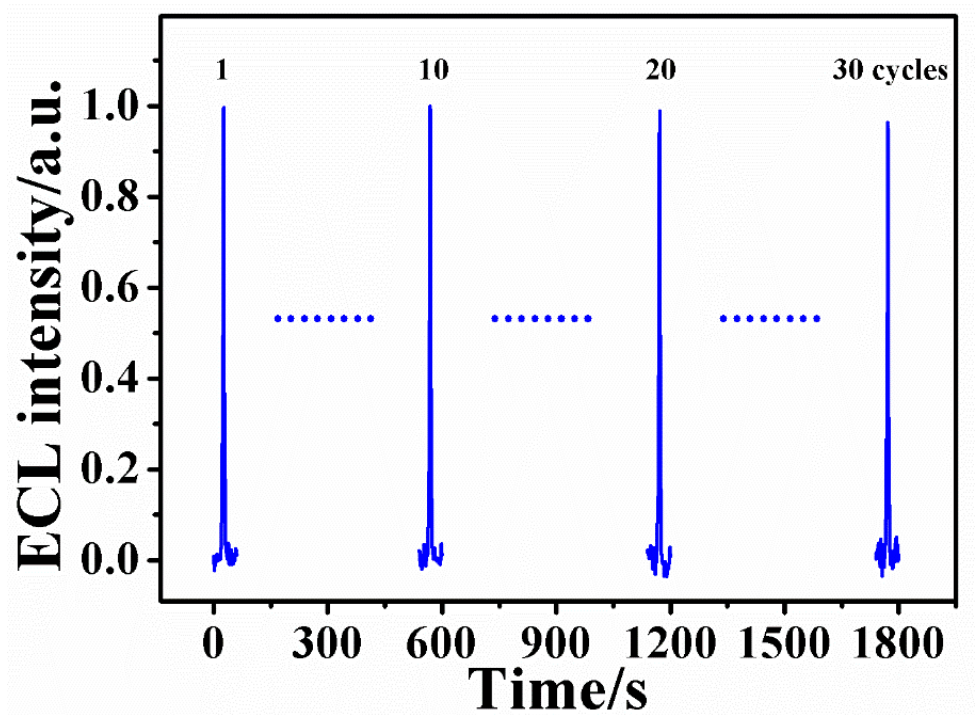

Figure S16. ECL emission of the $(\mathrm{CDs} @ \mathrm{PSS} / \mathrm{LDH})_{36}$ UTF modified ITO electrode with continuous cyclic voltammetry $(\mathrm{CV})$ for 30 cycles.
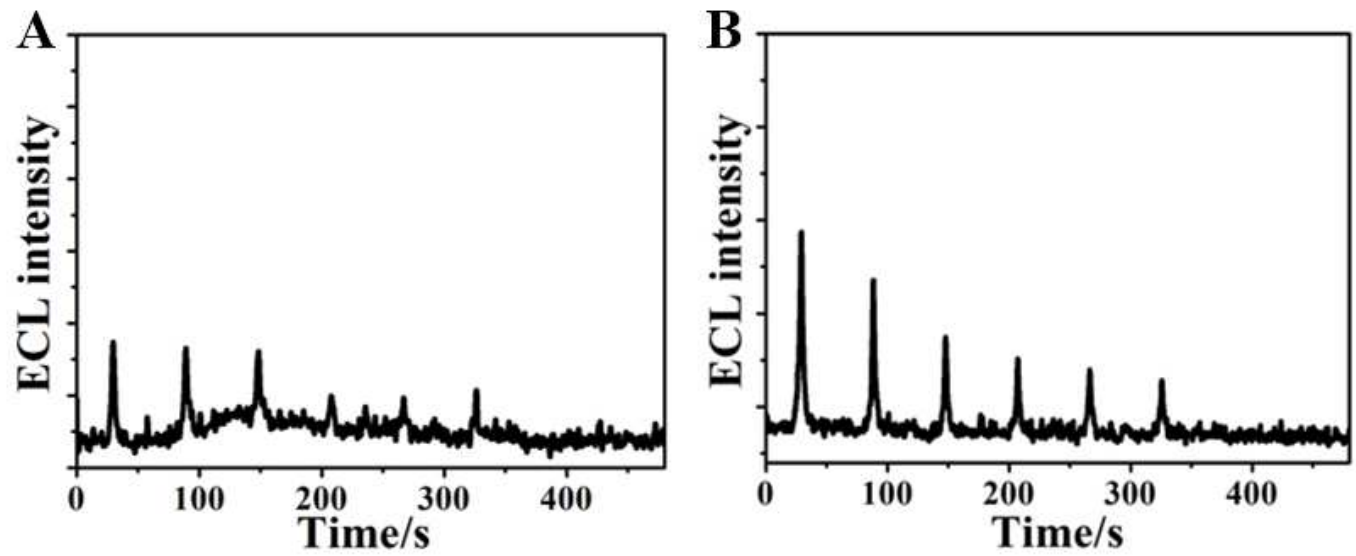

Figure S17. ECL signals of (A) CDs drop-casted ITO electrode and (B) CDs@PSS drop-casted ITO electrode. 


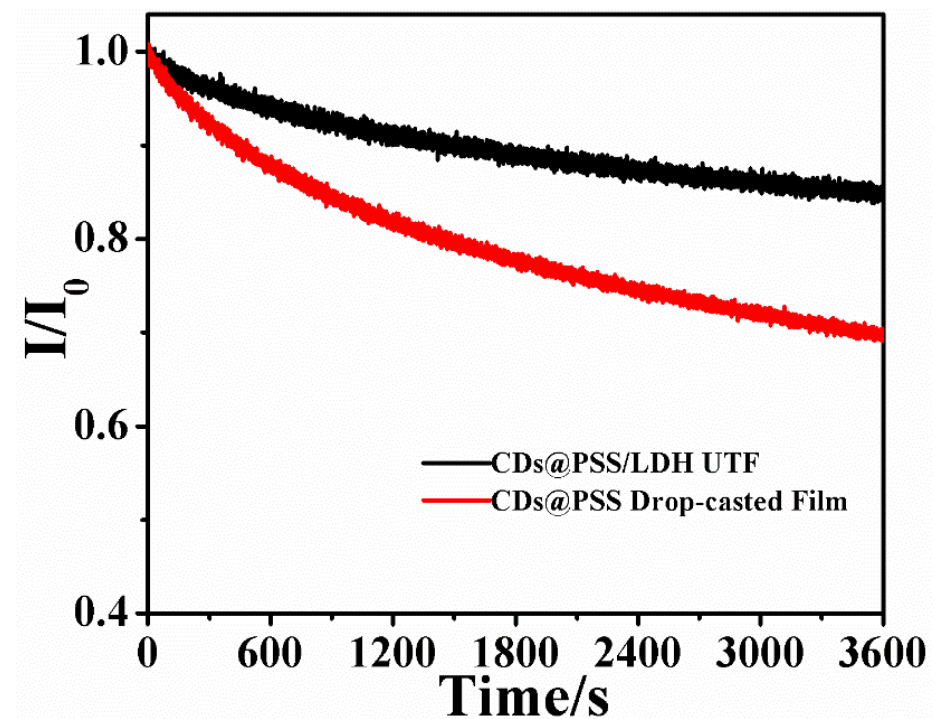

Figure S18. Photostability of the (CDs@PSS/LDH) $)_{36}$ UTF and CDs@PSS drop-casted film upon irradiation by UV light $(370 \mathrm{~nm})$ for $60 \mathrm{~min}$.

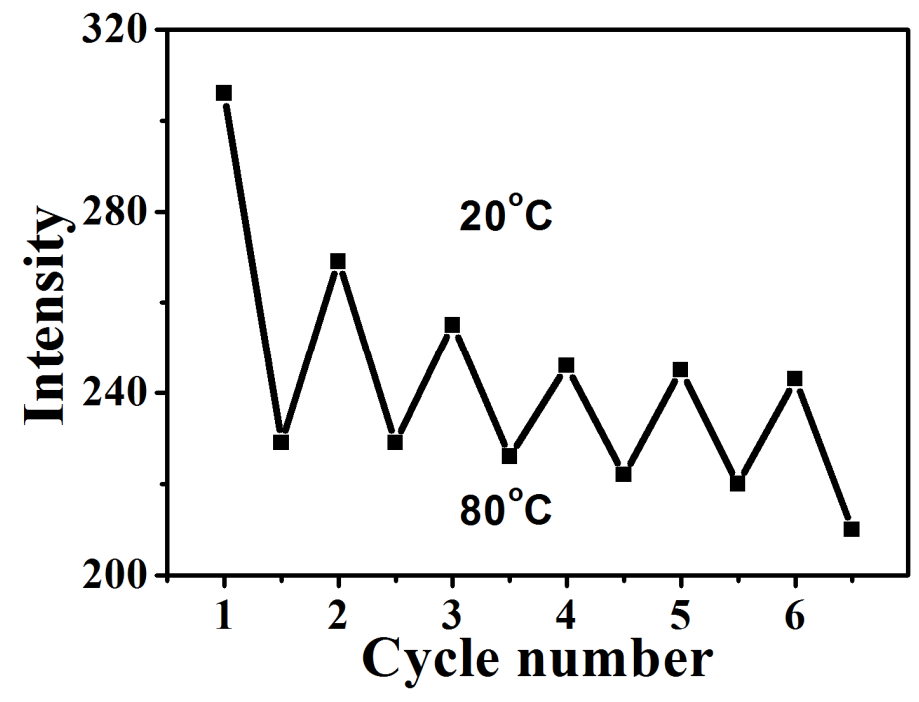

Figure S19. Photoluminescence stability of the CDs@PSS drop-casted film in the temperature range $20-80^{\circ} \mathrm{C}$. 


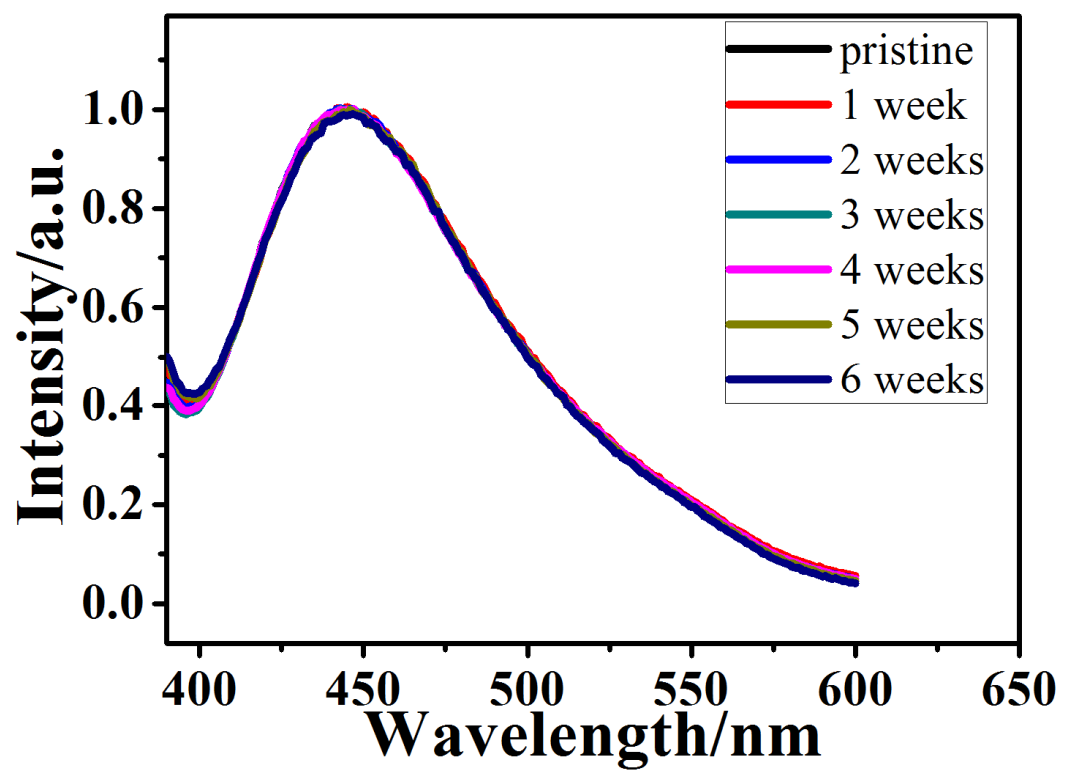

Figure S20. Fluorescence spectra of (CDs@PSS/LDH) $)_{36}$ UTF recorded within six weeks. 


\section{Reference}

(1) Zhu, S.; Meng, Q.; Wang, L.; Zhang, J.; Song, Y.; Jin, H.; Zhang, K.; Sun, H.; Wang, H.; Yang, B. Highly Photoluminescent Carbon Dots for Multicolor Patterning, Sensors, and Bioimaging. Angew. Chem. Int. Ed. 2013, 52, 3953-3957.

(2) Liang, R.; Xu, S.; Yan, D.; Shi, W.; Tian, R.; Yan, H.; Wei, M.; Evans, D. G.; Duan, X., CdTe Quantum Dots/Layered Double Hydroxide Ultrathin Films with Multicolor Light Emission Via Layer-by-Layer Assembly. Adv. Funct. Mater. 2012, 22, 4940-4948.

(3) Gupta, V.; Chaudhary, N.; Srivastava, R.; Sharma, G. D.; Bhardwaj, R.; Chand, S. Luminscent Graphene Quantum Dots for Organic Photovoltaic Devices. J. Am. Chem. Soc. 2011, 133, 9960-9963.

(4) Segall, M. D.; Lindan, P. J. D.; Probert, M. J.; Pickard, C. J.; Hasnip, P. J.; Clark, S. J.; Payne, M. C. First-Principles Simulation: Ideas, Illustrations and the CASTEP Code. $J$. Phys.: Condens. Matter 2002, 14, 2717-2744.

(5) Payne, M. C.; Teter, M. P.; Allan, D. C.; Arias, T. A.; Joannopoulos, J. D. Iterative Minimization Techniques for Ab Initio Total-Energy Calculations: Molecular Dynamics and Conjugate Gradients. Rev. Mod. Phys. 1992, 64, 1045-1097.

(6) Perdew, J. P.; Burke, K.; Ernzerhof, M. Generalized Gradient Approximation Made Simple. Phys. Rev. Lett. 1996, 77, 3865-3868. 